\title{
A Dynamic Ice-Structure Interaction Model for Ice-Induced Vibrations by Using Van der Pol Equation
}

\author{
Xu Ji*, Erkan Oterkus** \\ * Department of Naval Architecture, Ocean and Marine Engineering University of Strathclyde \\ 100 Montrose Street Glasgow G4 0LZ, United Kingdom \\ Phone: +44 (0)141 5484094 \\ e-mail: x.ji@strath.ac.uk \\ ** Department of Naval Architecture, Ocean and Marine Engineering University of Strathclyde \\ 100 Montrose Street Glasgow G4 0LZ, United Kingdom \\ Phone: +44 (0)141 5483876 \\ e-mail: erkan.oterkus@strath.ac.uk
}

\begin{abstract}
A dynamic single degree-of-freedom ice-structure interaction model is developed based on a novel physical mechanism combination between self-excited vibration and forced vibration. Van der Pol equation, together with ice stress-strain rate curve and ice-velocity failure length are coupled to model the internal fluctuating nature of ice force in conjunction with the relative velocity caused by the structure as an external effect. Three distinct structural responses, quasi-static, steady-static and random modes have been captured during ice-structure interaction. And the key behaviour, ice force frequency lock-in phenomenon during ice-induced vibrations (IIV) is also observed. General results show good agreement with those in full-scale and small-scale experiments as well as in numerical models.
\end{abstract}

Keywords: Ice-structure interaction, ice-induced vibrations, Van der Pol equation, frequency lock-in, resonance

\section{Nomenclature}

\begin{tabular}{|ll|}
\hline$\varepsilon$ & Strain rate $\left(\mathrm{s}^{-1}\right)$ \\
$v_{r}$ & Relative velocity between ice and structure $(\mathrm{m} / \mathrm{s})$ \\
$v_{i}$ & Ice velocity $(\mathrm{m} / \mathrm{s})$ \\
$x$ & Structural velocity $(\mathrm{m} / \mathrm{s})$ \\
$\lambda$ & Dimensionless coefficient \\
$D$ & Structural diameter $(\mathrm{m})$ \\
$\sigma$ & Ice strength $\left(\mathrm{N} / \mathrm{m}^{2}\right)$ \\
$\sigma_{\max }$ & Maximum stress at ductile-brittle range $\left(\mathrm{N} / \mathrm{m}^{2}\right)$ \\
$\sigma_{d}, \sigma_{b}$ & Minimum stress at ductile and brittle range $\left(\mathrm{N} / \mathrm{m}^{2}\right)$ \\
$\alpha, \beta$ & Positive and negative indices to control the envelope profile \\
$v_{t}$ & Transition ice velocity approximately in the middle of transition range $(\mathrm{m} / \mathrm{s})$ \\
$f_{i}$ & Ice failure frequency $(\mathrm{Hz})$ \\
\hline
\end{tabular}




\begin{tabular}{|c|c|}
\hline$L$ & Ice failure length $(\mathrm{m})$ \\
\hline$c$ & Ratio of $v_{i}$ and $L$ \\
\hline$M$ & Mass (kg) \\
\hline$K$ & Structural stiffness $(\mathrm{N} / \mathrm{m})$ \\
\hline$C$ & Damping $(\mathrm{kg} / \mathrm{s})$ \\
\hline$X$ & Structural displacement $(\mathrm{m})$ \\
\hline$X$ & Structural velocity $(\mathrm{m} / \mathrm{s})$ \\
\hline$X$ & Structural acceleration $\left(\mathrm{m} / \mathrm{s}^{2}\right)$ \\
\hline$F$ & Ice force $(\mathrm{N})$ \\
\hline$T$ & Time $(s)$ \\
\hline$\alpha$ & Magnification factor calibrate from experiment data \\
\hline$q$ & Dimensionless fluctuation variable \\
\hline$a, \varepsilon$ & Scalar parameters that control the $q$ profile to behave like a real ice force \\
\hline$\omega_{i}$ & Angular frequency of ice force $(\mathrm{rad} / \mathrm{s})$ \\
\hline$b$ & Coefficient depending on ice properties \\
\hline$Y$ & Ice displacement (m) \\
\hline$Y$ & Ice velocity $(\mathrm{m} / \mathrm{s})$ \\
\hline$Y$ & Ice acceleration $\left(\mathrm{m} / \mathrm{s}^{2}\right)$ \\
\hline$t$ & Normalized time \\
\hline$x$ & Normalized structural displacement \\
\hline$l$ & Normalized ice failure length \\
\hline$y$ & Normalized ice velocity \\
\hline$\xi$ & Damping ratio \\
\hline$\omega_{n}$ & Angular natural frequency of structure $(\mathrm{rad} / \mathrm{s})$ \\
\hline$\tau$ & Reduced angular structural frequency \\
\hline$U_{r}$ & Reduced ice velocity \\
\hline$F_{\max }$ & Maximum ice force at $\sigma_{\max }(\mathrm{N})$ \\
\hline$f_{s}$ & Predominant structural response frequency $(\mathrm{Hz}$ \\
\hline
\end{tabular}

\section{Introduction}

Offshore structures subjected to the action of drifting ice floes may experience several kinds of interactions with the ice. For instance, columns fixed to the seabed are commonly used as a form of ice-resistant offshore structures. Sometimes, this kind of structures experiences sustained interaction with the ice, which is called as ice-structure interaction, and severe vibration may occur under certain velocities (Yue and Guo, 2011). These vibrations, known as ice-induced vibrations (IIV), would lead to fatigue problems, along with safety issues and uncomfortable working and living conditions. 
Reasons of IIV proposed by researchers can be divided into two categories; negative damping (Blenkarn, 1970; Määttanen, 1981) and resonance (Sodhi, 1988). Ice force frequency lock-in, when ice force frequency is strongly dominated by structural natural frequency, exists in both of these explanations (Määttänen, 1983; Wang and Xu, 1991; Huang and Liu, 2009; Yue and Guo, 2011). Both these two types of IIV are considered as self-excited vibration by the majority and on the opposite of this mechanism is the forced vibration. It has been very debatable that whether resonance is either self-excited vibration or forced vibration for almost 50 years, claimed by Määttänen and Sodhi, respectively. Määttänen (2015) further mentioned that if the structure is not flexible enough, there should not be any self-excited vibration for the resonance caused by frequency lock-in. On the other hand, Sodhi (1988) discussed this as forced vibration because ice force still exists even when the structure is stopped from moving, which is supported by Timoshenko and Young (1937) as well as definitions from Den Hartog (1947):

- In self-excited vibration, the alternating force that sustains the motion is created or controlled by the motion itself. When the motion stops, the alternating force disappears.

- In forced vibration, the sustaining alternating force exists independently apart from the motion and persists even when the vibratory motion is stopped.

From ice point of view, in forced vibration model, ice has its own failure characteristic and is not associated with either structural properties or structural motion variables. On the other hand, in self-excited vibration, ice force is controlled by not only ice itself, but also structural motions. Since it is a fact that ice has its own characteristics, structural properties or motion variables will definitely be involved when ice is in contact with a structure and ice failure occurs. So, regardless of the structure being rigid or flexible, wide or narrow, vertical or conical, the main issue is how strong these structural effects can be added to the original ice failure behaviour. This is the reason why forced vibration or self-excited vibration mechanisms are predominant from time to time.

Many effective single degree-of-freedom ice-structure interaction models have been developed since 1960s. It is a difficult process because of various ice properties, lack of data and unclear ice failure mechanism. Matlock et al. (1971) proposed the revolutionary idea that ice breaks into a certain size and the model can capture key structural responses under low and high velocities. However, ice force frequency is too chaotic to capture, even though it has the steady-state structural response under intermediate velocities. As Matlock et al. (1971) mentioned, this model is incapable of capturing resonance behaviour since periodic force-time function is missing. In addition, ice resists the synchronization or frequency lock-in between structure and ice, when structure moves backwards from the maximum displacement. On the other hand, Wang and $\mathrm{Xu}$ (1991) utilized the Van der Pol equation as an ice force oscillator to simulate periodic as well as self-excited characteristics. Only structural response frequency lock-in phenomenon has been captured which is different than the ice force frequency lock-in that Huang and Liu (2009) proposed. In this study, a single degree-of- 
freedom ice-structure interaction model, ice force oscillator model is developed based on a novel physical mechanism combination between self-excited vibration and forced vibration. Van der Pol equation, together with ice stress-strain rate curve and ice-velocity failure length are utilized to model the internal fluctuating nature of ice force in conjunction with the relative velocity caused by structure as an external effect.

\section{Mechanical Features of Ice Crushing}

To simulate or predict a proper result, an approximate equivalent forcing term is applied to the equation of motion, which makes each numerical model different from each other (Matlock et al., 1971; Wang and Xu, 1991; Sodhi, 1995; Huang and Liu, 2009). Different ice failure properties can cause different distinctive forces either on frequency or amplitude under different velocities (Blenkarn, 1970; Sodhi and Haehnel, 2003; Timco and Johnston, 2004; Kärnä, 2007) .

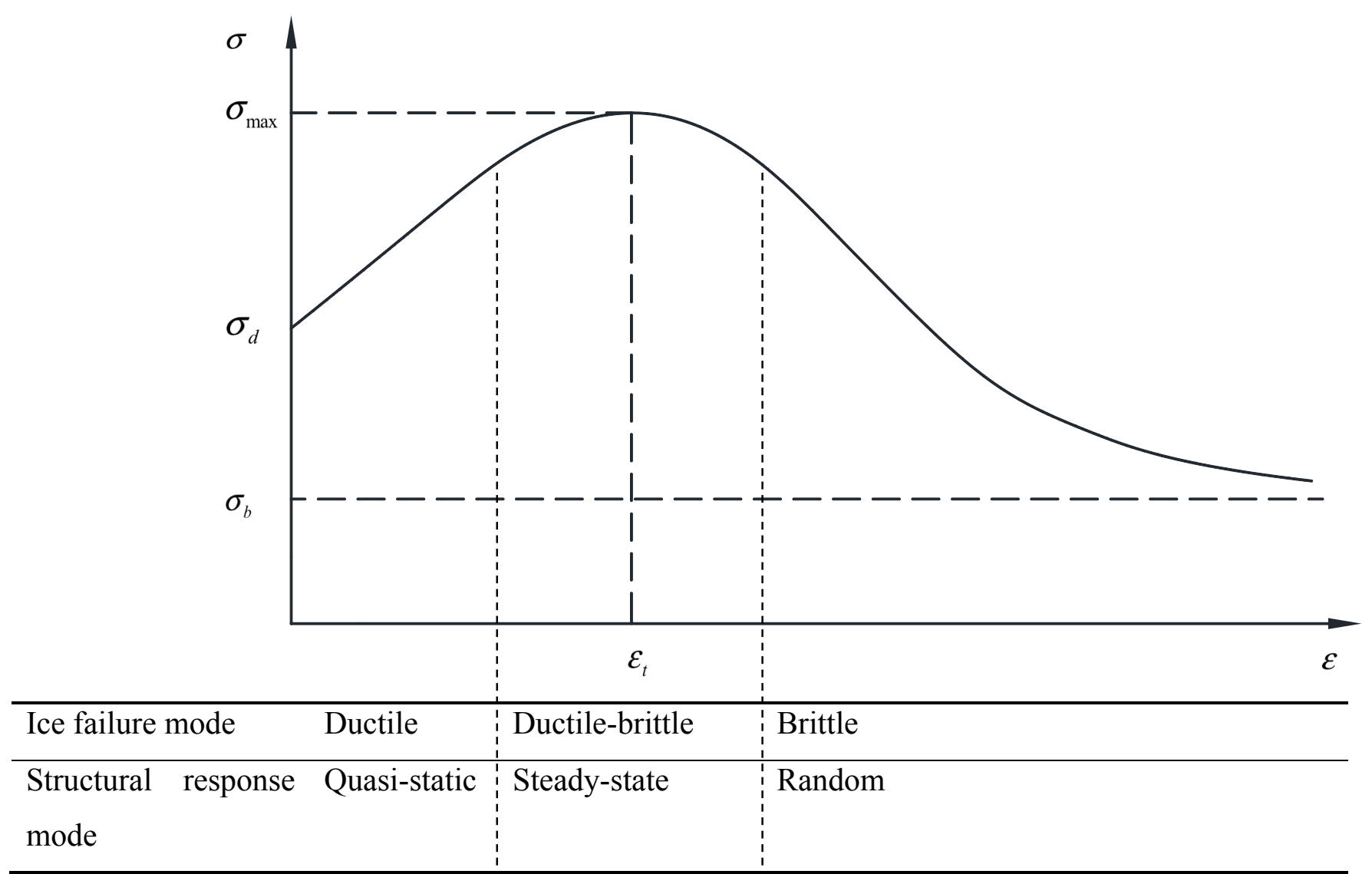

Fig.1 Strain rate vs. uniaxial or indentation stress corresponding to ice failure and structural response mode

\subsection{Ice Stress-Strain Rate Curve}

It is found and proved that ice uniaxial strength or indentation strength is a function of strain rate as shown in Fig.1 (Blenkarn, 1970; Michel and Toussaint, 1978; Palmer et al., 1983; Sodhi and Haehnel, 2003). The strain rate is defined by $v_{r} / \lambda D$, where the dimensionless coefficient $\lambda$ varies from 1 to 4 and $D$ is the structural diameter (Yue and Guo, 2011). Based on the dimensionless numerical relationship between the 
stress and strain rate (Huang and Liu, 2009), it can be expressed by two separate dimensional exponential functions:

$$
\sigma= \begin{cases}\left(\sigma_{\max }-\sigma_{d}\right)\left(v_{r} / v_{t}\right)^{\alpha}+\sigma_{d}, & v_{r} / v_{t} \leq 1 \\ \left(\sigma_{\max }-\sigma_{b}\right)\left(v_{r} / v_{t}\right)^{\beta}+\sigma_{b}, & v_{r} / v_{t}>1\end{cases}
$$

where $\sigma_{\max }$ is the maximum stress at ductile-brittle range, $\sigma_{d}$ and $\sigma_{b}$ are the minimum stress at ductile and maximum stress brittle range, respectively, $\alpha$ and $\beta$ are positive and negative indices to control the envelope profile, respectively, and $v_{t}$ is the transition ice velocity approximately in the middle of transition range.

\subsection{Ice Failure Length}

Observations show that ice fails in wedge shape after one loading phase with a characteristic frequency at both full-scale and small-scale experiments. During the loading phase, micro-cracks inside the ice accumulate. When the density of micro-cracks reaches a critical level, ice is incapable of taking more load, leading to a coalescence of ice cracks in a whole amount, which is called unloading phase (Yue and Guo, 2011). Kärnä et al. (1993) used high speed camera and observed that ice fails at a certain amount after each loading phase. Neill (1976) reviewed numerous data and emphasized that ice fails by following a certain size distribution and ice velocity controls the ice failure frequency. Sodhi and Morris (1986) conducted small-scale tests pushing different diameter rigid cylinders at different velocities against ice sheets with different thicknesses, and found that the ice failure frequency is strongly proportional to ice velocity and slightly inversely proportional to diameter, and proposed that

$$
f_{i}=c \frac{v_{i}}{H}
$$

where $f_{i}$ is the ice failure frequency, $H$ is the ice thickness. $c$ is the ratio of the ice thickness to ice failure length $L$, i.e. $c=H / L$, and it ranges from 2 to 5, with an average value of around 3.Tong et al. (2001) conducted another similar series of small-scale tests and found the same trend. Palmer et al. (2010) reviewed both full-scale and small-scale data and proposed that the dimensionless velocity-thickness ratio parameter is more consistent than velocity-diameter ratio parameter to define different structural response modes.

\subsection{Similarity with vortex-induced vibrations}

As a result of the ice failure mechanism described in the previous section, ice-induced vibrations is often discussed as analogous to vortex-induced vibrations (Johansson, 1981; Palmer et al., 2010). The fluctuating nature of vortex shedding is similar to that of ice failure, causing periodic force upon structure. Furthermore, the similarity between vortex-shedding spacing and ice failure length leads to the analogical frequency calculation to Eq. (2) (Sodhi, 1988), in which vortex-shedding frequency $f_{v}$ can be defined as 
$f_{v}=S t \cdot v_{f} / D$, where $S t$ is the Strouhal number describing the oscillating flow mechanism and $v_{f}$ is the vortex shedding frequency.

\section{Model Description}

As shown in Fig.2, the structure is modelled as a mass-spring-damper system. Ice is moving towards the structure at a constant velocity. Ice force is calculated by area times stress. Ice failure is controlled by a periodic force-time oscillator, Van der Pol equation, which is used to model the saw-tooth shape of ice force fluctuation profile to demonstrate its internal natural failure behaviour, coupling the relative velocity $v_{r}$ to correlate the strain energy into ice during both loading and unloading phases (Wang and $\mathrm{Xu}, 1991$ ). Apart from utilization of the ice stress-strain rate relationship given in Eq. (1), another main advantage of the current approach is that the relative displacement between ice and structure is also considered during computations because compressive stress will transfer to ice deformation. When the deformation exceeds the natural ice failure length, ice will also fail, overcoming the overlook of structural effect on ice failure in Wang and Xu's model. So, in addition to the Van der Pol oscillator, ice will also fail instantaneously when the relative displacement is larger than ice failure length, $L$. Therefore, ice will fail under both internal and external effects.

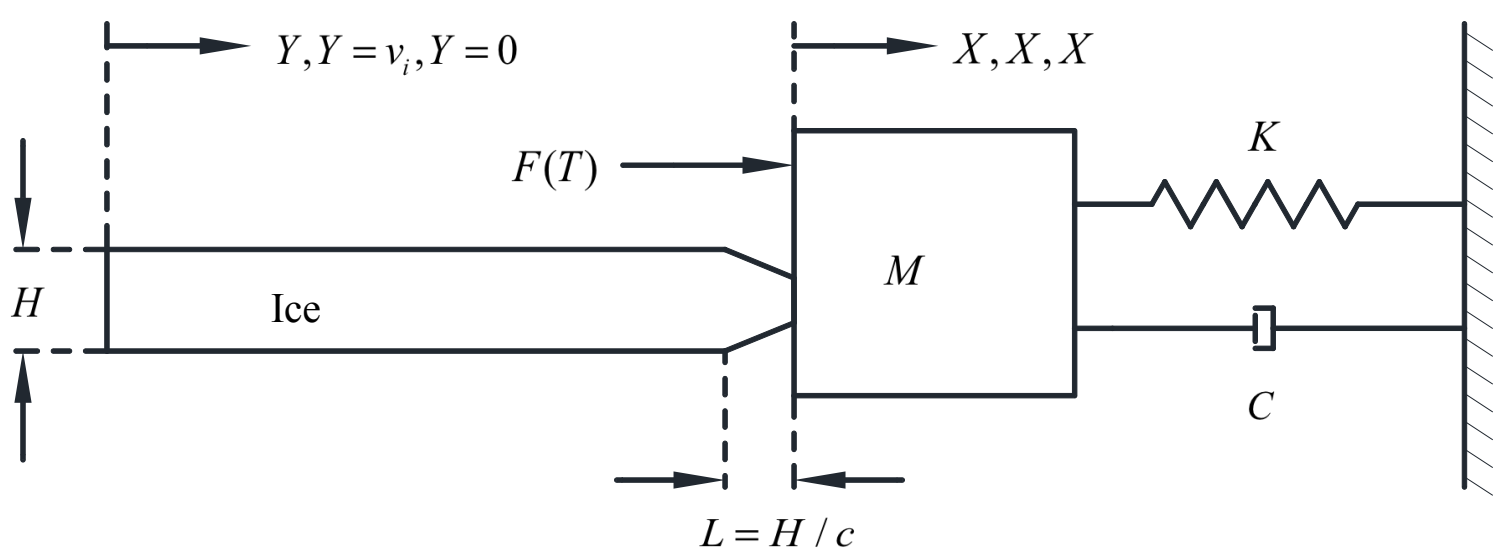

Fig.2 Schematic sketch of dynamic ice-structure model

Therefore, this single degree-of-freedom ISI model can be represented by using three equations. These are equation of motion and Van der Pol equation, i.e.

$$
\left\{\begin{array}{l}
M X+C X+K X=F(T)=\alpha D H \sigma(q+a) \\
q+\varepsilon \omega_{i}\left(q^{2}-1\right) q+\omega_{i}^{2} q=\frac{b \omega_{i}}{H}(Y-X)
\end{array}\right.
$$

in conjunction with the ice stress-strain rate equation given in Eq. (1). In Eq. (3), $X$ is the displacement of the structure, the "dot" symbol represents the derivative with respect to time $T, \alpha$ is the magnification factor obtained from experiment data, $D$ is the structural diameter, $q$ is the dimensionless fluctuation 
variable, $a$ and $\varepsilon$ are scalar parameters that control the $q$ profile to behave like a real ice force, $\omega_{i}=2 \pi f_{i}$ is the angular frequency of ice force, $b$ is a coefficient depending on ice properties and $Y$ is the displacement of ice. Introducing the normalized quantities: $t=T \omega_{i}, x=X / H, l=L / H, y=Y /\left(\omega_{i} H\right)$, Eq. (3) can be transformed into dimensionless form as

$$
\left\{\begin{array}{l}
x+2 \xi \tau x+\tau^{2} x=\frac{\alpha D \sigma \tau^{2}}{K}(q+a) \\
q+\varepsilon\left(q^{2}-1\right) q+q=b(y-x)
\end{array}\right.
$$

where $\xi=C /\left(2 M \omega_{n}\right)$ is the damping ratio, $\omega_{n}=\sqrt{K / M}$ is the angular natural frequency of structure, $\tau=\omega_{n} / \omega_{i}$ is the reduced angular structural frequency, and lead to the reduced ice velocity $U_{r}$ as

$$
\tau=\frac{\omega_{n}}{2 \pi c\left(v_{i} / H\right)}=\frac{1}{c U_{r}}, U_{r}=\frac{2 \pi}{\omega_{n}} \frac{v_{i}}{H}=\frac{v_{i}}{f_{n} H}
$$

When $\omega_{i}$ is close to $\omega_{n}$, synchronization will occur defining a lock-in situation. Moreover, when $\tau=1$ at $U_{r}=1 / c$, a perfect lock-in will occur.

\section{Dynamic Behaviour}

To date, studies have mainly focused on the ice velocity effect on ice force and structural dynamic behaviour. Coupled system configuration parameters are determined from the tests described in Huang et al. (2007) and specified as:

- $\quad$ Adopted from Huang et al. (2007)

$D=0.076 \mathrm{~m}, \omega_{n}=39.27 \mathrm{rad} / \mathrm{s}, K=27.44 \mathrm{kN} / \mathrm{m}, H_{1}=0.048 \mathrm{~m}$

- Adjusted or assumed

$H_{2}=0.024 \mathrm{~m}, \xi=0.15, \alpha=0.06, a=2, \varepsilon=2.5, b=0.1, \sigma_{\max }=100 \mathrm{kPa}, \sigma_{d}=70 \mathrm{kPa}, \sigma_{b}=50 \mathrm{kPa}$, $v_{t}=0.15 \mathrm{~m} / \mathrm{s}$

During the steady-state vibration, the maximum structural velocity is found to have approximately a linear relationship with the ice velocity in many experiments (Kärnä, 2001). Fig.3 shows a good match for both the amplitude and the different structural response mode under different ice velocities. Steady-state vibration range in the test (black line) is from $0.11 \mathrm{~m} / \mathrm{s}$ to $0.205 \mathrm{~m} / \mathrm{s}$ and from $0.08 \mathrm{~m} / \mathrm{s}$ to $0.22 \mathrm{~m} / \mathrm{s}$ in the model (red line), which is determined from the corresponding ice force frequency lock-in range in Fig.5 (red line). On the left and right side of this range are quasi-static mode and random response mode, respectively. Following this linear relationship, Kärnä and Trunen (1990) found that there are different upper limit and 
velocity range for different ice thicknesses according to the field records. Therefore, in another configuration, ice thickness is considered to be $H_{2}=0.024 \mathrm{~m}$, which is the half of the original thickness. Results for this case (blue line) are shown in Fig.3. In this case, maximum structural velocity reduces by half because half thickness reduces compressed area by half leading to half loading force. In addition, ice failure length also decreases by half leading to ice failure frequency twice higher and makes the resonance or ice force frequency lock-in range twice earlier, which is from $0.04 \mathrm{~m} / \mathrm{s}$ to $0.11 \mathrm{~m} / \mathrm{s}$ as opposed to from $0.08 \mathrm{~m} / \mathrm{s}$ to $0.22 \mathrm{~m} / \mathrm{s}$ for $H_{1}=0.048 \mathrm{~m}$. The variations in amplitude and range agree well with Kärnä and Trunen's observations, i.e. scaling down the behaviour observed in the original case to approximately one half.

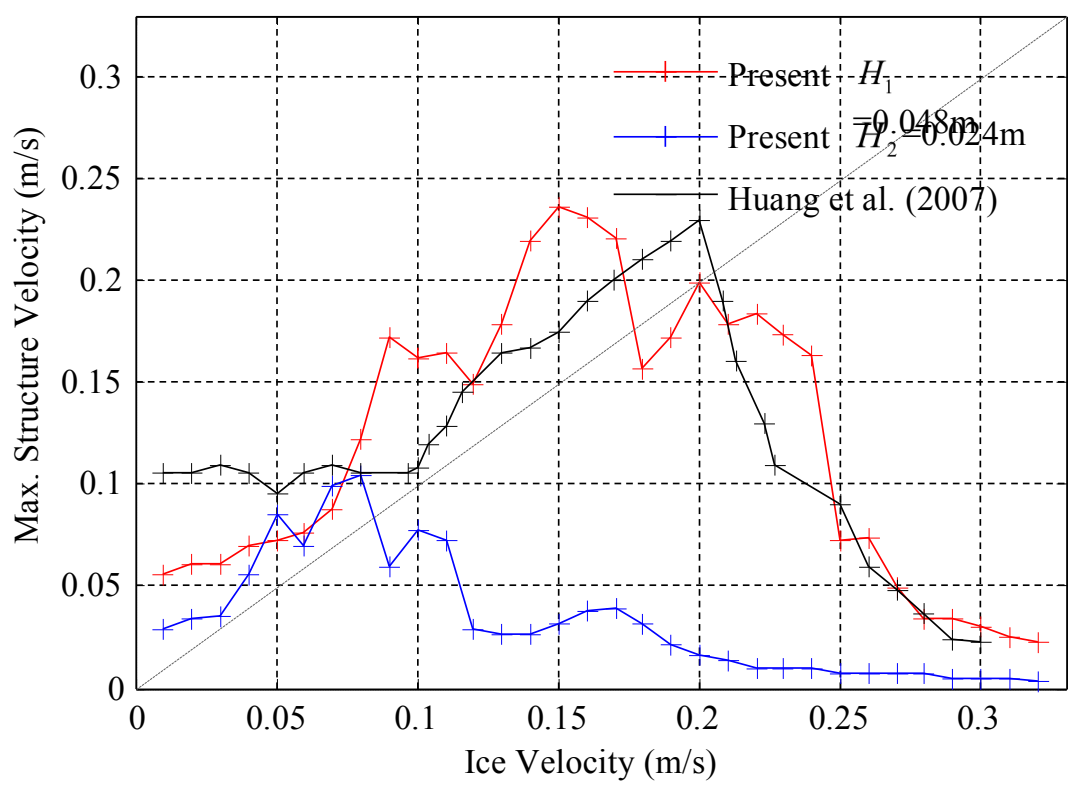

Fig.3 Ice velocity vs. the maximum structural velocity 

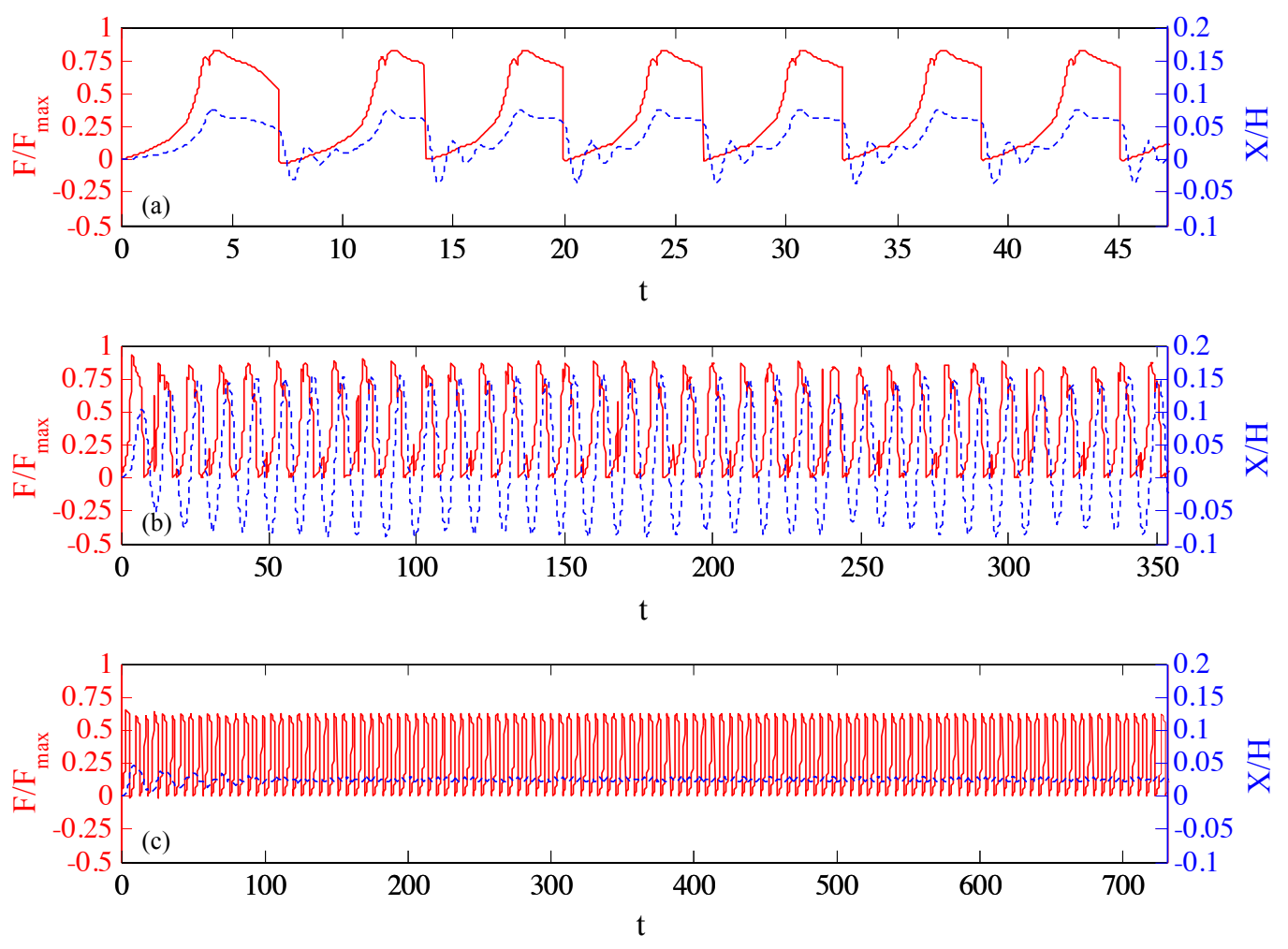

Fig.4 Normalized time vs. normalized structural displacement (blue dash line) and normalized force (red line) for the ice thickness value of $H_{1}=0.048 \mathrm{~m}$ : (a) Quasi-static at $U_{r}=0.07$; (b) Steady-state at $U_{r}=0.50$ and (c) Random at $U_{r}=1.03$

To show the normalized ice force (red line) and structural response (normalized displacement, blue dash line) transition during time histories, three representative velocities are picked from three distinctive structural response mode regions, as shown in Fig.4, where $F_{\max }=0.10944 \mathrm{kN}$ is the maximum ice force at the maximum stress. Both of them captured the key characteristic behaviours observed in experimental tests done by Huang et al. (2007). Moreover, overall behaviour, i.e. saw tooth profile, sinusoidal structural response, and how the ice force and structural response change with ice velocity, are in good agreement both qualitatively and in most cases quantitatively with what many researchers cited in this paper including Matlock et al. (1971) and Kärnä (2001). In quasi-static region, shown in Fig. 4 (a), ice sheet fails in bending with ductile behaviour where a slow loading to the maximum is then followed by a quick unloading. At this stage, ice force and structural displacement are in phase with each other.

When ice speed increases to a critical value corresponding to the transitional ice velocity at the lower limit of the ice force frequency lock-in range as shown in Fig. 4(b), where ice fails between bending and crushing with ductile-brittle behaviour, loading and unloading takes less time. In addition to the ice internal natural failure behaviour defined in Eq. (2), failure as a result of the real-time ice-structure external effect is more 
significant, in which sudden unloading takes place sometimes due to the fact that relative displacement exceeds the tolerable ice failure length. Consequently, ice force frequency will stay in this range leading to a resonance condition for a fairly large range as Yue and Guo (2011) observed in the field, where ice force and structural displacement are 90 degrees out-of-phase. In other words, ice and structure are moving in the same direction and structure is accelerated by the ice during loading phase. During the unloading phase, ice and structure are moving in the opposite direction. Moreover, the structure is decelerated by the ice as well since ice deformation will cause failure to occur when the compressive stress exceeds a condition that the natural failure length can tolerate.

When ice speed exceeds another critical value corresponding to the transitional ice velocity at the upper limit of the ice force frequency lock-in range, ice will fail as crushing with brittle behaviour, in which structure responds at small amplitude with no obvious predominant frequency, as shown in Fig.4 (c) and Fig.6, respectively. Furthermore, ice force amplitude at intermediate velocity range is around $40 \%$ higher than that at higher speed as the contribution from coupled ice stress-strain rate curve, which aligns with the $40 \%$ ice loading difference due to velocity effect between intermediate and high velocities (Timco and Johnston, 2004).

\section{Ice-Induced Vibration Phenomenon}

Ice force frequency lock-in is the significant phenomenon during IIV process. By transforming the timehistory forces to spectrum analysis using fast Fourier transform method, the predominant ice force frequencies under two different ice thicknesses are shown in Fig.5. Two lines at different thicknesses are following almost exactly the same pattern after the ice velocity normalised to reduced velocity, especially for the frequency locking in range, from $U_{r}=0.267$ to 0.733 when $H_{1}=0.048 \mathrm{~m}$ and from $U_{r}=0.3$ to 0.7 when $H_{2}=0.024 \mathrm{~m}$. This range matches well with the range suggested by Palmer et al. (2010) varying from 0.01 to 0.4 and to 0.8 occasionally. Moreover, most of the lock-in frequencies are slightly lower than the structural natural frequency that can also be confirmed from Kärnä et al. (2013). On the left and right side of this region are quasi-static and random modes, in which the ice force frequency in these two ranges is following a linear relationship with the ice velocity, and the slope is equal to $c$, as defined in Eq. (2). This similar lock-in range prediction can also be found in Huang and Liu (2009), whereas there is no need to define the initial conditions specifically in the present model and system will adjust to stable conditions automatically. 


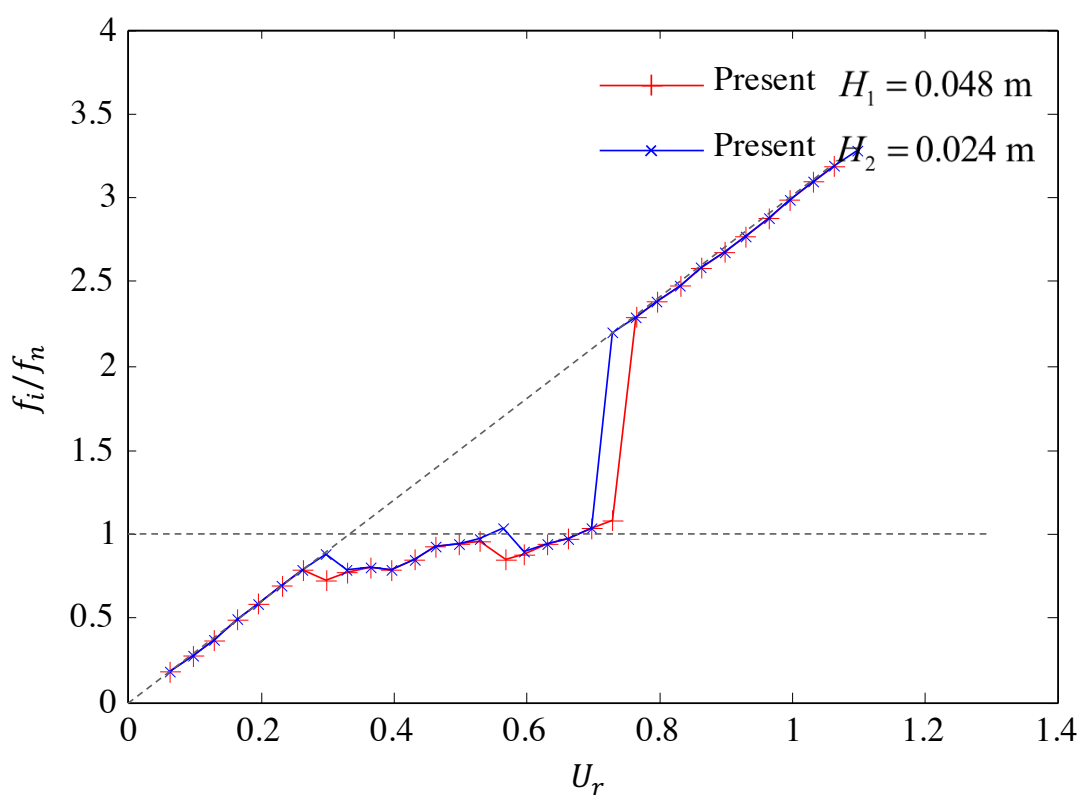

Fig.5 Reduced velocity vs. ratio between predominant ice force frequency $f_{i}$ and structural natural frequency $f_{n}$

Accordingly, the predominant frequency of the structural response increases with the velocity till the lock-in range, as shown in Fig. 6. Then, it fluctuates under the natural frequency of the structure following a similar trend with that in Fig. 5, and stays at around the natural frequency afterwards. This relationship can also be confirmed from experimental results obtained by Määttänen (1983) and Tsuchiya et al. (1985), and numerical model result generated from Matlock et al. (1971) model. However, structural response frequency has not only its value, but also its corresponding response amplitude at the same time, indicating the structural response amplitude. The colour bar in Fig. 6 shows the relative amplitude difference (relative amplitude is based on the maximum response value at $U r=0.5$ ), from which it shows the structural response increasing at quasi-static region then vibrating at high response as well as reaching the maximum during steady-state range. As the velocity increases further, frequency value stays at around the natural frequency while the response amplitude is decreasing to almost zero, which can also be verified from Huang et al. (2007). 


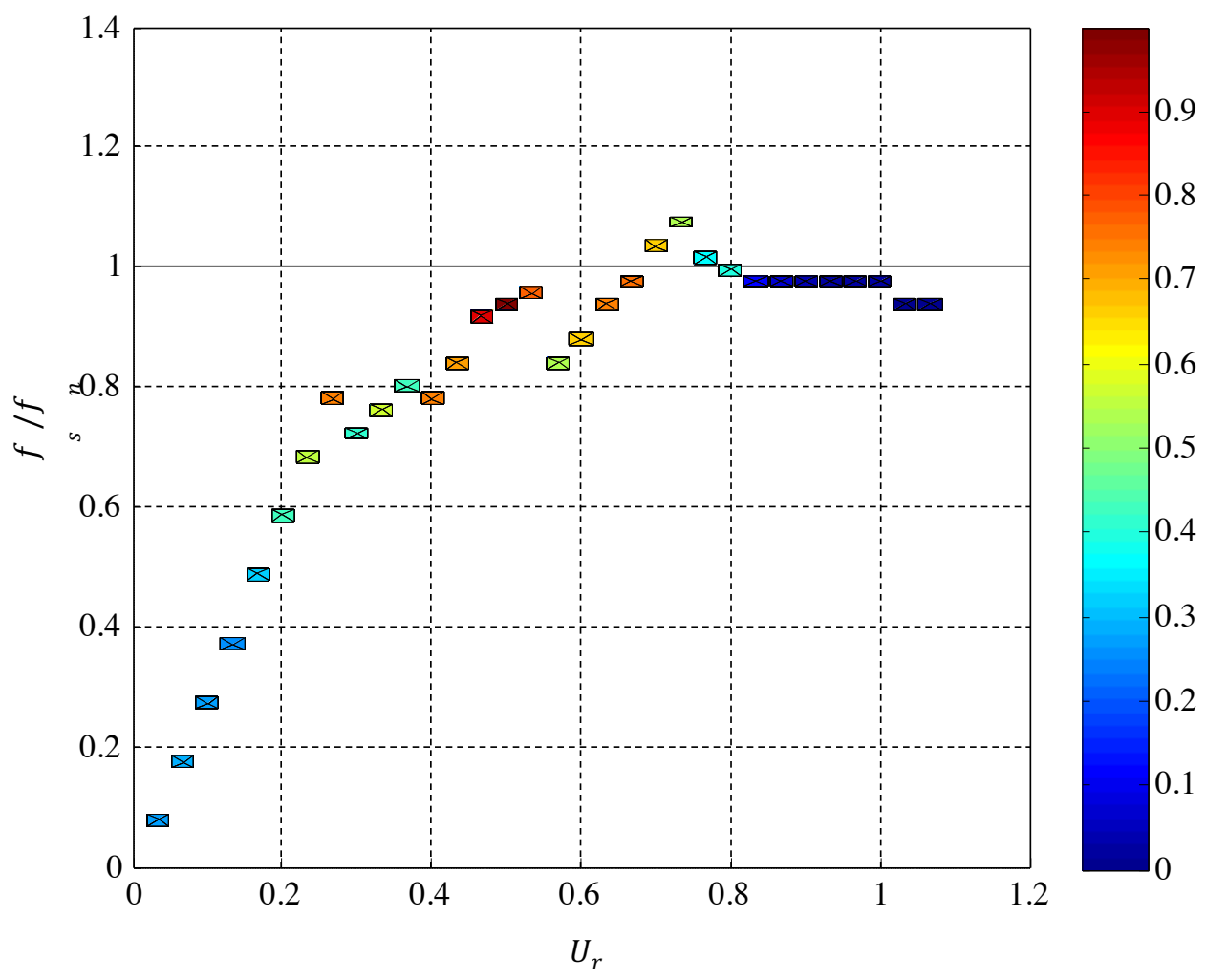

Fig.6 Reduced velocity vs. ratio between predominant structural response frequency $f_{s}$ and structural natural frequency $f_{n}, H_{1}=0.048 \mathrm{~m}$

\section{Conclusion}

In this study, the physical mechanism in ice-induced vibrations between forced vibration and self-excited vibration and the similarity between ice-induced vibrations and vortex-induced vibrations are discussed. On the basis of ice behaviour observed in full and small scale experiments, a novel dynamic ice-structure interaction numerical model is developed considering ice stress variations as well as its internal and external effects. Results show good agreements with that in full and small scale experiments and that in other numerical models, like Matlock et al. (1971) and Huang and Liu (2009), including structural velocity relationship with ice velocity, ice force and structural displacement profile for three distinct modes from low to high velocities, and the IIV phenomenon caused by ice force frequency lock-in.

\section{Acknowledgements}

The first author would like to acknowledge the University Research Studentship provided by University of Strathclyde and Dr. Narakorn Srinil for useful discussions. 


\section{References}

BLENKARN, K. Measurement and analysis of ice forces on Cook Inlet structures. Offshore Technology Conference, 1970. Offshore Technology Conference.

DEN HARTOG, J. 1947. Mechanical Vibrations, New York, McGraw-Hill.

HUANG, G. \& LIU, P. 2009. A dynamic model for ice-induced vibration of structures. Journal of Offshore Mechanics and Arctic Engineering, 131, 011501.

HUANG, Y., SHI, Q. \& SONG, A. 2007. Model test study of the interaction between ice and a compliant vertical narrow structure. Cold Regions Science and Technology, 49, 151-160.

JOHANSSON, P. 1981. ICE-INDUCED VIBRATION OF FIXED OFFSHORE STRUCTURES, PART 1: REVIEW OF DYNAMIC RESPONSE ANALYSES.

KÄRNÄ, T. Simplified modelling of ice-induced vibrations of offshore structures. 16th International Symposium on Okhotsk Sea \& Sea Ice, 4-8 February 2001 Mombetsu, Japan. 114-122.

KÄRNÄ, T. Research Problems Related to Time-Varying Ice Actions. Proceedings of the International Conference on Port and Ocean Engineering Under Arctic Conditions, 2007.

KÄRNÄ, T., ANDRESEN, H., GURTNER, A., METRIKINE, A., SODHI, D., LOO, M., KUIPER, G., GIBSON, R., FENZ, D. \& MUGGERIDGE, K. Ice-Induced Vibrations of Offshore StructuresLooking Beyond ISO 19906. 22nd International Conference on Port and Ocean Engineering Under Arctic Conditions (POAC), Espoo, Finland, 2013. 1673-1684.

KÄRNÄ, T., MUHONEN, A. \& M., S. Rate effects in brittle ice crushing. Conf. Port Ocean Eng. under Arctic Conditions. (POAC'93), August 1993 Hamburg. 59-71.

KÄRNÄ, T. \& TRUNEN, R. A straightforward technique for analyzing structural response to dynamic ice action. Proceedings of the Ninth International Conference on Offshore Mechanics and Arctic Engineering (OMAE), 1990. 135-142.

MÄÄTTANEN, M. 1981. Laboratory tests for dynamic ice-structure interaction. Engineering Structures, 3, 111-116.

MÄÄTTÄNEN, M. 1983. Dynamic ice-structure interaction during continuous crushing. DTIC Document.

MÄÄTTÄNEN, M. Ice induced frequency lock-in vibrations - Converging towards consensus. Proceedings of the International Conference on Port and Ocean Engineering under Arctic Conditions, POAC, 2015.

MATLOCK, H., DAWKINS, W. P. \& PANAK, J. J. 1971. Analytical model for ice-structure interaction. Journal of the Engineering Mechanics Division, 97, 1083-1092.

MICHEL, B. \& TOUSSAINT, N. 1978. Mechanisms and theory of indentation of ice plates. Journal of glaciology, 19, 285-300.

NEILL, C. R. 1976. Dynamic ice forces on piers and piles. An assessment of design guidelines in the light of recent research. Canadian Journal of Civil Engineering, 3, 305-341.

PAlmer, A., GOODMAN, D., ASHBY, M., EVANS, A., HUTCHINSON, J. \& PONTER, A. 1983. Fracture and its role in determining ice forces on offshore structures. Annals of glaciology, 4, 216221.

PALMER, A., QIANJIN, Y. \& FENGWEI, G. 2010. Ice-induced vibrations and scaling. Cold Regions Science and Technology, 60, 189-192.

SODHI, D. S. Ice-induced vibrations of structures. Proceedings of the Ninth International Association of Hydraulic Engineering and Research Symposium on Ice, 1988. 625-657.

SODHI, D. S. 1995. An ice-structure interaction model. Studies in Applied Mechanics, 42, 57-75.

SODHI, D. S. \& HAEHNEL, R. B. 2003. Crushing ice forces on structures. Journal of cold regions engineering, 17, 153-170.

SODHI, D. S. \& MORRIS, C. E. 1986. Characteristic frequency of force variations in continuous crushing of sheet ice against rigid cylindrical structures. Cold Regions Science and Technology, 12, 1-12.

TIMCO, G. \& JOHNSTON, M. 2004. Ice loads on the caisson structures in the Canadian Beaufort Sea. Cold Regions Science and Technology, 38, 185-209.

TIMOSHENKO, S. \& YOUNG, D. 1937. Vibration Problems in Engineering, (1937). D van Nostrand Company, Inc., New-York.

TONG, J., SONG, A. \& SHI, Q. 2001. An experimental study of ice-induced vibration and dynamic characteristics of ice loads. Ocean Eng, 19, 34-39. 
TSUCHIYA, M., KANIE, S., IKEJIRI, K., IKEJIRI, A. \& SAEKI, H. An experimental study on icestructure interaction. Offshore Technology Conference, 1985. Offshore Technology Conference.

WANG, L. \& XU, J. Description of dynamic ice-structure interaction and the ice force oscillator model. 11 th International Conference on Port and Ocean Engineering under Arctic Conditions- POAC'91, St. John's, Newfoundl, Can, 09/24-28/91, 1991. 141-154.

YUE, Q. \& GUO, F. 2011. Physical Mechanism of Ice-Induced Self-Excited Vibration. Journal of Engineering Mechanics, 138, 784-790. 